\title{
Role of NDP- and FZD4-Related Novel Mutations Identified in Patients with FEVR in Norrin/ $\beta$-Catenin Signaling Pathway
}

\author{
Shuai Han $\mathbb{D}^{1,2,3,4}$ Junhui Sun $\mathbb{D}^{1,3}$ Liwei Yang $\mathbb{D}^{2}$, and Ming Qi $\mathbb{D}^{1,3,4,5,6}$ \\ ${ }^{1}$ Department of Cell Biology and Medical Genetics, School of Medicine, Zhejiang University, Hangzhou 310000, China \\ ${ }^{2}$ Department of Obstetrics, Zhejiang Provincial People's Hospital, People's Hospital of Hangzhou Medical College, No. 158, \\ Shangtang Road, Hangzhou, Zhejiang Province, China \\ ${ }^{3}$ Zhejiang California International Nanosystems Institute, Zhejiang University, Hangzhou 310000, China \\ ${ }^{4}$ DIAN Diagnostics, Hangzhou 310000, China \\ ${ }^{5}$ Assisted Reproduction Unit, Department of Obstetrics and Gynecology, Department of Laboratory Medicine, Sir Run Run \\ Shaw Hospital, Zhejiang University School of Medicine, Key Laboratory of Reproductive Dysfunction Management of \\ Zhejiang Province, Hangzhou 310000, China \\ ${ }^{6}$ Department of Pathology and Laboratory Medicine, University of Rochester Medical Center, Rochester, New York 14642, USA
}

Correspondence should be addressed to Ming Qi; mingqi@zju.edu.cn

Received 23 January 2020; Accepted 2 March 2020; Published 27 April 2020

Academic Editor: Kazuhisa Nishizawa

Copyright (C) 2020 Shuai Han et al. This is an open access article distributed under the Creative Commons Attribution License, which permits unrestricted use, distribution, and reproduction in any medium, provided the original work is properly cited.

\begin{abstract}
Mutations in NDP and FZD4 have been closely related to a series of retinal diseases including familial exudative vitreoretinopathy (FEVR). Our study was designed to identify novel NDP and FZD4 mutations by whole exome sequencing (WES) in a cohort of patients with a definitive diagnosis of FEVR and explore the underlying molecular mechanism. During 2016, we investigated fifty nonconsanguineous families with affected individuals exhibiting FEVR phenotype and WES identified one recently reported mutation: NDP c.127C>A (p.H43N), and five novel mutations: NDP c.129_131del (p.44del), NDP c.320_353del (p.R107Pfs), NDP c.321delG (p.L108Cfs), NDP c.377G > T (p.C126F), and FZD4 c.314T > G (p.M105R) that cosegragated with the abnormal fundus vascular manifestations in six families. All the mutations were perceived to be pathogenic or likely pathogenic according to the standards and guidelines from the American College of Medical Genetics and Genomics (ACMG) and predicted to be deleterious by a series of bioinformatics analyses. We systematically performed functional analyses on the six mutations utilizing the Topflash reporter assay, where all NDP and FZD4 mutants revealed at least 50\% loss of wild-type activity. Immunoprecipitation finally demonstrated that the six mutations could degrade the Norrin-Frizzled-4 pair-binding effect to varying degrees. Finally, our study underscores the correlation between the FEVR phenotype and genotype in NDP and FZD4, extending the mutation spectrum, allowing a reliable assessment of FEVR recurrence and improving genetic counseling. Further, our findings provide essential evidence for the follow-up study of animal models and drug targets by Topflash assays and immunoprecipitation.
\end{abstract}

\section{Introduction}

Familial exudative vitreoretinopathy (FEVR) is a rare inheritable retinal-vascular disorder characterized by retinal avascularity and first described by Criswick and Schepens in 1969 [1]. Typically, the visual problems of FEVR have been featured by secondary complications such as macular and vascular dragging, tractional retinal detachment, retinal folds, ocular neovascularization, and vitreous hemorrhage $[2,3]$.
The diverse expressivity of FEVR could be demonstrated as intrafamilial variability and disease asymmetry [4-9].

To our knowledge, numerous investigations have been conducted to date into the pathological mechanism of FEVR, where the canonical Wnt signaling network has been demonstrated to play a pivotal role during retinal organogenesis and angiogenesis $[6,10]$. The Norrie disease protein (NDP), Norrin, an unconventional Wnt ligand, coordinated with coreceptors: Frizzled-4 (FZD4), LRP5, and TSPAN12, has been 
elucidated to induce the Norrin/ $\beta$-catenin canonical signaling pathway, one of the main drivers for retinal angiogenesis in the mammalian eye $[6,11-15]$. As Xu et al. described, Norrin with a cysteine-knot motif could bind specifically with the cysteine-rich domain (CRD) of Frizzled-4 and potently activate the classical Wnt signaling pathway [13]. Thus, mutations of NDP and FZD4 in cysteine-knot motif and CRD, respectively, are a good model for elucidating the high affinity of the Norrin-Frizzled-4 pair. Mutations in NDP have been associated with X-linked FEVR (MIM\#305390), and mutant FZD4 is linked to an autosomal dominant form of FEVR (MIM\#133780). The similar clinical phenotype presented by NDP and FZD4 mutants has also led us to inquire the mechanism of the NorrinFrizzled-4 pair involved in retinal vasculogenesis.

In our study, we successfully detected one reported mutant and five novel mutants of NDP and FZD4 in fifty Chinese patients with FEVR during 2016 by whole exome sequencing (WES). Then, we showed that FEVR-associated Norrin and Frizzled-4 mutations inhibited the canonical signaling cascade and the possible mechanism: the decrement of Norrin-Frizzled-4 pair affinity, which might implicate the significant phenotypic effect of the patients.

\section{Materials and Methods}

2.1. Patient Screening. Fifty patients, who underwent professional ophthalmology examinations and diagnosed as FEVR, were performed WES of peripheral blood genomic DNA during 2016 in Sir Run Run Shaw Hospital, Zhejiang University School of Medicine. Only FEVR patients with novel mutations of NDP and FZD4 were gathered at the time of study initiation. Informed consent was obtained from all participants in the study. This study adhered to the tenets of the Declaration of Helsinki on human subjects and was approved by School of Medicine, Zhejiang University, Hangzhou, China.

2.2. Next Generation Sequencing, Data Analysis, and Mutation Validation. Genomic DNA samples were extracted from peripheral blood leukocytes using a QIAGEN QIAamp DNA Blood Mini Kit (Qiagen, Hilden, Germany), subjected to whole-exome capture on Agilent SureSelect Human All Exon V6 Capture (Agilent, California, USA) and performed high-throughput sequencing on Illumina HiSeq 2000 (Illumina, Inc., San Diego, CA, USA). The sequence data were aligned to the human reference genome: University of California, Santa Cruz (UCSC) hg19 (http://genome.ucsc.edu) with Burrows-Wheeler aligner version 0.7.10: BWA-MEM (http://biobwa.sourceforge.net/). Further, we calibrated variants using the Genomic Analysis Toolkit (https://software .broadinstitute.org/gatk/) and conducted functional annotation by Annovar (http://www.openbioinformatics.org/ annovar/) and SnpEff (http://www.snpeff.sourceforge.net). The benign variants were filtered with minor allele frequency $(\mathrm{MAF})>1 \%$ in the 1000 Genomes data set (1000G) (https:// www.internationalgenome.org/1000-genomes-browsers/), the Single Nucleotide Polymorphism (dbSNP) (http://www.ncbi .nlm.nih.gov/SNP.), Genome Aggregation Database (gno-
mAD) (http://gnomad.broadinstitute.org/), Exome Aggregation Consortium (ExAC) (http://exac.broadinstitute.org/), and our internal database. Human Gene Mutation Database (HGMD) (http://www.hgmd.cf.ac.uk/ac/index.php), ClinVar (http://www.ncbi.nlm.nih.gov/clinvar/), Online Mendelian Inheritance in Man (OMIM) (https://OMIM.org), and Leiden Open Variation Database (LOVD) (http://lovd.nl) were used to annotate the existence of mutation reports. The silico analyses of the missense variants were predicted by PolyPhen-2 (http://genetics.bwh.harvard.edu/pph2/), Sorting Intolerant From Tolerant (http://sift-dna.org), and Mutation Taster (http://www.mutationtaster.org/). We performed Sanger sequencing to validate the suspicious variants and confirm the segregation of the identified variants in the affected and unaffected family members. PCR primers were designed as follows: NDP-127/129-131-F: TTCCTTGAA CGGGACTGGAT; NDP-127/129-131-R: AGCCTCATTCT CCCACAAG; NDP-320-353/321/377-F: GCAACGAGTGT GAGGGTCTT; NDP-320-353/321/377-R: CCCAAACAG CATTGAGAGCC; FZD4-314-F: AACTCAGCTTTGTGGG AGCA; FZD4-314-R: AATATGATGGGGCGCTCAGG.

2.3. 3D Modeling of NDP and FZD4 Missense Changes. We adopted University of California, San Francisco (UCSF) chimera software to mutate the residue identified in our study according to three-dimensional structure of Norrin and Frizzled-4 (PDB: 5pqc/5pqe).

2.4. Construction of Expression Plasmids. Wild-type NDP cDNA was purchased from Kelei Biological Technology Co., Ltd. (Shanghai, China) and amplified using forward 5' -CCGctcgagCGGATGAGAAAACATGTACTAGCTG-3' and reverse primer $5^{\prime}$-CGggatccCGGGAATTGCATTCCT CGCAGTGA- ${ }^{\prime}$. Then, we subcloned wild-type NDP cDNA into pEGFP-N1 vector (BD Biosciences) using XhoI and BamHI sites. All NDP mutants were introduced into the wild-type NDP cDNA by primer-mediated PCR mutagenesis. It should be noted that for deletion frameshift mutations (NDP c.321del/320_353del), in order not to affect the subsequent expression of EGFP, the reverse primer was designed as follows: $5^{\prime}$-CGggatccGGAATT GCATTCCTCGCAGTGA-3'.

The recombinant plasmids containing NDP-EGFP fusion constructs were verified by direct DNA sequencing and then amplified and purified for transfection (Qiagen Inc., Valencia, CA). Construction of wild-type and mutant pCMV-3xFlag-FZD4 recombinant plasmids was described as above using HindIII and BamHI sites. The corresponding primers were as follows: forward $5^{\prime}$-aagcttATGGCCTGGCG GGGCGCA- $3^{\prime}$ and reverse primer $5^{\prime}$-CggattcCGTACC ACAGTCTCACTG-3'.

The primers for site-directed mutagenesis were NDP127-mutant-f: TGCATGAGGCACaACTATGTGG ATT; NDP 127-mutant-r: ACGTACTCCGTGtTGATAC ACCTAA; NDP 129_131-mutant-f: CATGAGGCAC(cta)CATGTGGATTCTAT; NDP 129_131-mutant-r: GTACTC CGTGGT(gat)ACACCTAAG ATA; NDP 320_353-mutant-f: GCACTGC(...)CACCTACCGGTACATCCTCT; NDP $320_{-}$ 
131-mutant-r: CGGTAGGTG(...)GCAGTGCCTTCAGCTT GG; NDP 321-mutant-f: AGGCACTGCG(g) CTGCGA TGCT; NDP 321-mutant-r: TCCGTGACGC(c) GACGCT ACGA; NDP 377-mutant-f: CATCCTCTCCTtTCA CTGC GAG; NDP 377-mutant-r: GTAGGAGAGGaAGT GACG CTC; FZD4 314-mutant-f: TTTATGTGCCAAgGTGCAC AGAGAA; FZD4 314-mutant-r: TTCTCTGTGCACcTTGG CACATAAA.

2.5. Topflash Report Assay. To generate HEK 293 cell line harboring SuperTopFlash (STF) reporter gene, $125 \mathrm{ng}$ of the STF construct (Kelei Biological Technology Co., Ltd., Shanghai, China) and $125 \mathrm{ng}$ of pSV2neo (Kelei Biological Technology Co., Ltd., Shanghai, China) providing a neomarker for dominant selections screening out by $600 \mu \mathrm{g} / \mathrm{ml}$ of G418 (Invitrogen Corporation, Carlsbad, California, USA) were stably transfected into HEK 293 cells in a 48-well plate using PolyJet (Invitrogen Corporation, Carlsbad, California, USA) [16]. The HEK 293 cell lines harboring STF reporter gene were cotransfected with $200 \mathrm{ng}$ of Norrin (wild type or mutant), $200 \mathrm{ng}$ of FZD4 (wild type or mutant), $200 \mathrm{ng}$ of LRP5, and $100 \mathrm{ng}$ of pSV- $\beta$-gal (Kelei Biological Technology Co., Ltd., Shanghai, China) in a 48-well plate using PolyJet (Invitrogen Corporation, Carlsbad, California, USA). Fortyeight hours after transfection, we washed the cells twice with $1 \mathrm{x}$ PBS and subjected to luciferase assay using Dual Luciferase assay reagent (Promega, USA).

2.6. Western Blotting. Western blotting was performed as described previously using $\beta$-catenin monoclonal antibody (1: 1000, Beyotime AF0069) [17].

2.7. Generation of Norrin-Conditioned Medium. HEK 293T cells were seeded at a density of $0.8 \times 10^{6}$ in six $10 \mathrm{~cm}^{2}$ plates and transfected with mutant or wild-type NDP-EGFP fusion constructs. Sixteen hours later, the medium was removed and replaced with fresh medium. After thirty-six hours, the medium was neutralized by adding sterile $1 \mathrm{M}$ HEPES $\mathrm{pH} 8.0$ and conditioned for additional one day. The medium was concentrated in Amicon Ultra 3K Millipore.

2.8. Native Polyacrylamide Gel Electrophoresis. Plasma Membrane Protein Isolation and Cell Fractionation Kit (Invent Biotechnologies, Inc., Eden Prairie, USA) was used to isolated membrane proteins. A NativePAGE Preparation Kit (Sangon Biotech, Shanghai, China) was adopted to make up a standard $8 \%$ polyacrylamide gel, which was loaded (without SDS and reducing reagent) with $8 \mu \mathrm{l}$ of corresponding conditioned medium for each construct tested. Electrophoresis was carried out using as described by Qin et al. [18].

2.9. Immunoprecipitation. We transfected HEK 293T cells from a $60 \mathrm{~mm}^{2}$ plate with wild-type FZD4-3xFlag constructs or mutants, which were subsequently incubated with $3 \mathrm{ml}$ of the mutant or wild-type Norrin conditional medium on ice for one hour. Subsequent immunoprecipitation was performed using GFP monoclonal antibody $(1: 1000$, Beyotime AF0159) and Flag monoclonal antibody (1:1000, Beyotime AF5051) as described by Xu et al. [13].

\section{Results}

3.1. Novel Mutations Identified in NDP and FZD4 and Bioinformatics Prediction. Six novel mutations of NDP and FZD4 were identified by WES from six unrelated FEVR probands among fifty nonconsanguineous patients, validated by Sanger sequencing during 2016 (Figure 1). However, in the performance of the following functional experiments, NDP c.127C >A (p.H43N) had been reported by Rao et al. in May 2017 [19]. All mutations were proved to be cosegregated with the abnormal phenotype of FEVR probands and perceived as pathogenic or likely pathogenic in accordance with the American College of Medical Genetics and Genomics (ACMG) guidelines, where NDP c.127C>A (p.H43N), NDP c.129_131del (p.44del), NDP c.320_353del (p.R107Pfs), NDP c.321delG (p.L108Cfs), and FZD4 c.314T>G (p.M105R) were graded as pathogenic and NDP c.377G $>$ T (p.C126F) was graded as likely pathogenic (Table 1) [20]. Protein sequence alignment of human Norrin/Frizzled-4 with homologues from human and other species indicated that NDP p.H43N, NDP p.C126F, and FZD4 p.M105R were nonconservative substitutions (Figure S1). NDP p.H43N, NDP p.C126F, and FZD4 p.M105R resided in the Norrin-Frizzled-4 binding area and at the protein interface (Figures 2(a)-2(f)). Notably, NDP p.C126F was involved in the second disulfide bond of cysteine knot structure, which were one of the highly conserved trio of disulfide bonds, so any other amino acid at this position would severely hinder the formation of the knot (Figures 2(h) and 2(j)) [21].

3.2. Defective Norrin/ $\beta$-Catenin Signaling. To verify the effect of the six mutations on the Norrin/ $\beta$-catenin signaling, a Wnt-responsive Topflash reporter assay was performed to elucidate the activation of canonical Norrin/ $\beta$-catenin signaling. As a result, all NDP and FZD4 mutants revealed at least $50 \%$ loss of wild-type activity (72.5\% for NDP p.H43N, 70\% for NDP p.44del, 71.2\% for NDP p.R107Pfs, $70.5 \%$ for NDP p.L108Cfs, $73 \%$ for NDP p.C126F, and $65 \%$ for FZD4 p.M105R) (Figures 3(a) and 3(b)). The expression of $\beta$ catenin was also detected after the transfection of the corresponding wild-type and mutant plasmids to HEK 293T cells, where the obvious reduction was also consistent with the FEVR presentations (Figures 3(c) and 3(d)). The attenuation of the activity of the Norrin/ $\beta$-catenin signaling strongly proved that the six mutations were pathogenic.

3.3. Defective Norrin and Frizzled-4 Binding. Norrin and Frizzled-4 are a high-affinity ligand-receptor pair, which could be used to isolate each other from the plasma membrane [13]. Norrin harbors the cysteine knot domain, which is cysteine-rich and involved in extracellular signaling transduction. Five NDP-related mutations were located in the domain, where most reported pathogenic mutations also resided (Figure 4(a)). Moreover, the specificity of NorrinFrizzled-4 binding has been assessed systematically, and the CRD of Frizzled- 4 is the only domain which Norrin binds to. FZD4 p.M105R was located in the CRD functional domain (Figure 4(a)). To investigate the mechanism of 


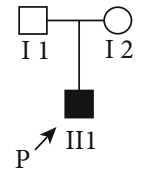

Family 1
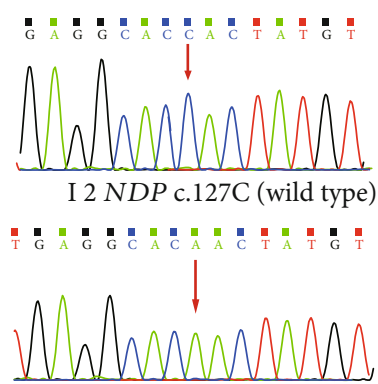

II 1 NDP c.127C>A(hemizygous)

(a)

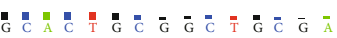

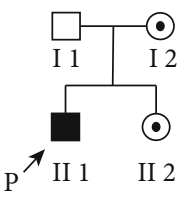

Family 3

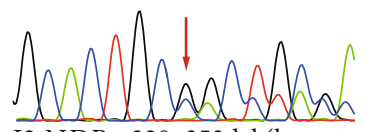

I2 NDP c.320_353del (heterozygous)

G

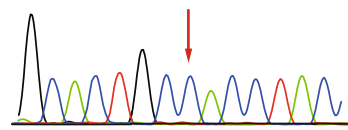

II1 NDP c.320_353del (hemizygous)

(c)

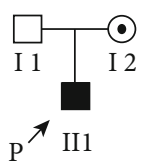

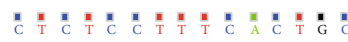

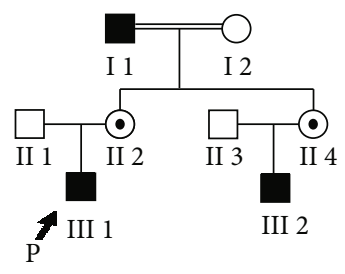

Family 5
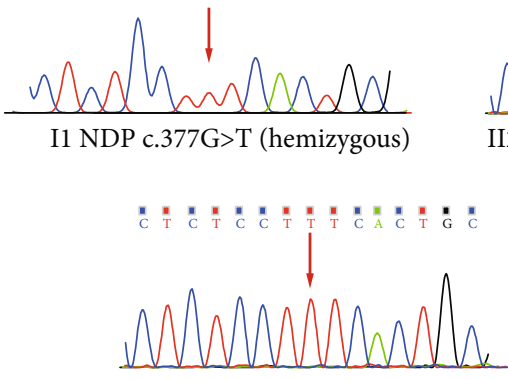

III1 NDP c.377G>T (hemizygous)
Family 4

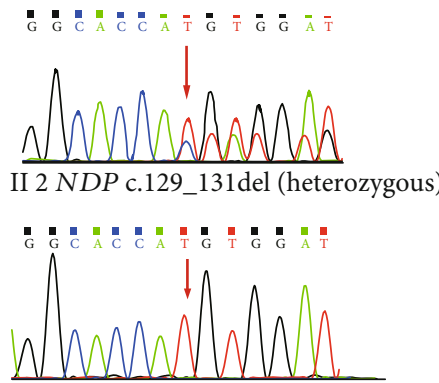

IV1 NDP c.129_131del (hemizygous)

(b)

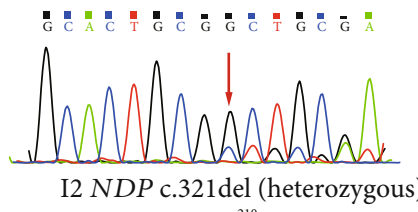

G G C A C G G C

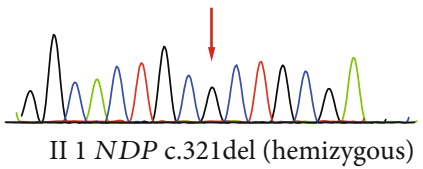

(d)

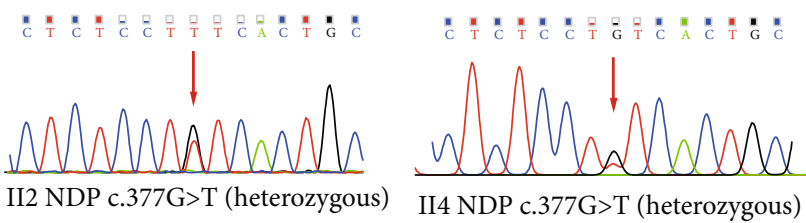

(e)

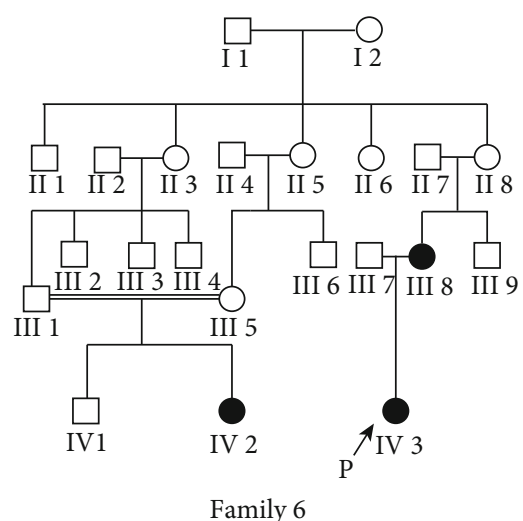

Family 6
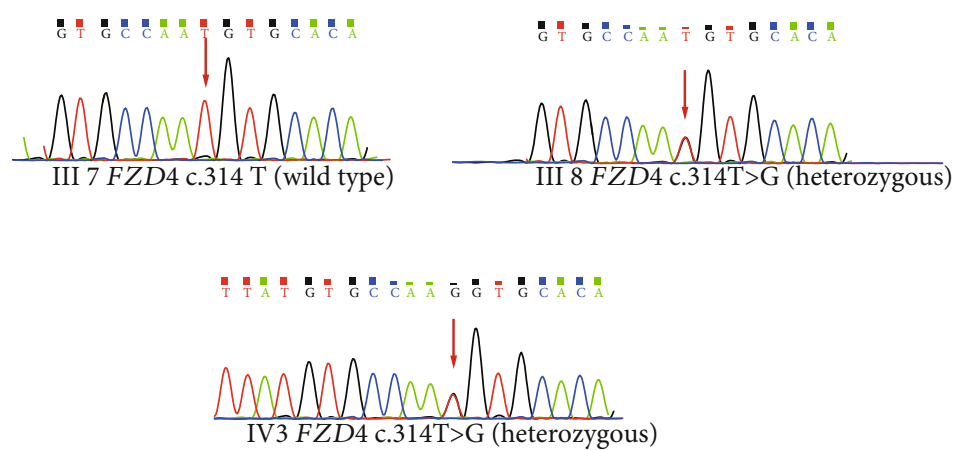

(f)

Figure 1: Pedigrees and DNA sequences. (a) Pedigree of family 1. The sequencing of the proband and his mother. (b) Pedigree of family 2. The sequencing of the proband and his mother. (c) Pedigree of family 3 . The sequencing of the proband and his mother. (d) Pedigree of family 4. The sequencing of the proband and his mother. (e) Pedigree of family 5 . The sequencing of the proband and his mother, aunt, grandfather, and cousin. (f) Pedigree of family 6 . The sequencing of the proband and his father and mother. The arrow showed the proband. The red arrow indicated the mutation. 
TABLE 1: Identified mutations of NDP and FZD4 in six families with FEVR.

\begin{tabular}{|c|c|c|c|c|c|c|c|c|}
\hline Family & Gene & Type & Mutation & $1000 \mathrm{G}$ & ExAC & gnomAD & Cosegregation & ACMG \\
\hline Family 1 & $N D P$ & Missense & c. $127 \mathrm{C}>\mathrm{A}, \mathrm{p} . \mathrm{H} 43 \mathrm{~N}$ & Novel & Novel & Novel & De novo & Pathogenic \\
\hline Family 2 & $N D P$ & Nonframeshift & c.129_131del, p.44del & Novel & Novel & Novel & Maternal & Pathogenic \\
\hline Family 3 & $N D P$ & Frameshift & c.320_353del, p.R107Pfs & Novel & Novel & Novel & Maternal & Pathogenic \\
\hline Family 4 & $N D P$ & Frameshift & c.321delG, p.L108Cfs & Novel & Novel & Novel & Maternal & Pathogenic \\
\hline Family 5 & $N D P$ & Missense & c.377G $>$ T, p.C126F & Novel & Novel & Novel & Maternal & Likely pathogenic \\
\hline Family 6 & FZD4 & Missense & c.314T>G, p.M105R & Novel & Novel & Novel & Maternal & Pathogenic \\
\hline
\end{tabular}

defective Norrin/ $\beta$-catenin signaling and further substantiate the interaction of Norrin and Frizzled-4 CRD binding, immunoprecipitation was performed. All NDP mutants bound Frizzled-4 at a severalfold lower level as determined by immunoblotting with anti-GFP and anti-Flag antibodies (Figures 4(b), 4(c), and 4(f)). Moreover, little mutant Frizzled-4 could precipitate with Norrin (Figures $4(\mathrm{~d})-4(\mathrm{f}$ )). Obviously, the binding effect was severely reduced, which correlated well with the extent of functional defects observed in the Topflash assays. Native polyacrylamide gel electrophoresis confirmed that the same extracellular secretion amount of mutant and wild-type Norrin was incubated with Frizzled4 and revealed a normal Frizzled-4 yield of the HEK 293T cytomembrane between the wild-type and mutant Frizzled4, indicating that the localization of mutant Frizzled-4 at plasma membrane was not affected (Figures $4(\mathrm{~g})-4(\mathrm{j})$ ).

\section{Discussion}

In our study, we identified one reported mutation: NDP c. $127 \mathrm{C}>\mathrm{A}(\mathrm{p} . \mathrm{H} 43 \mathrm{~N})$, and five novel FEVR-linked mutations: NDP c.129_131del (p.44del), NDP c.320_353del (p.R107Pfs), NDP c.321delG (p.L108Cfs), NDP c.377G $>$ T (p.C126F), and FZD4 c.314T $>$ G (p.M105R) by WES among fifty nonconsanguineous FEVR families during 2016 and excluded the presence of these mutations in 200 ethnically matched control individuals. It is critical to unravel the defective mechanism of FEVR-linked mutations identified in FEVR patients with genetic heterogeneity. Therefore, to assess if the missense mutations were important for Norrin/ $\beta$-catenin signaling, we first utilized bioinformatics tools to predict to be deleterious preliminarily. Finally, by Topflash assays and immunoprecipitation, we strongly proved that the Norrin and Frizzled-4 binding sensitivity of the six mutations was severely compromised and further impaired the Norrin/ $\beta$ catenin signaling, confirming the link between the mutants in NDP and FZD4 and abnormal retinal presentation.

Previous studies have demonstrated the variable impaired Norrin signal induced by different mutations observed regardless of the disease phenotype $[13,18,22$, 23]. Thus, the finding that the NDP and FZD4 mutations match the significant phenotypic effects could sufficiently evidence the presumed haploinsufficiency. It has been elucidated that NDP p.H43R and NDP H43Q are associated with Norrie (MIM\#310600), an inherited retinal disorder of highly overlapping ocular manifestations with FEVR [24, 25]. Notably, in our study, NDP p.H43N was found to be related to FEVR, which was a novel mutation when we con- ducted the research in 2016, was later reported by Rao et al. in May 2017, and was less characterized in the literature [19]. Thus, the reasons for different diseases caused by different substitutions of the amino acid at the same position also need to be explored. As our knowledge, Norrin function depends on three pairs of cysteines, forming the highly conserved disulfide bonds: $\mathrm{Cys}^{65}-\mathrm{Cys}^{126}$, $\mathrm{Cys}^{69}-\mathrm{Cys}^{128}$, and $\mathrm{Cys}^{39}{ }^{39} \mathrm{Cys}^{96}$, for which any substitution could possibly compromise signaling and Norrin-Frizzled-4 CRD binding, consistent with the significant decrement of signaling and binding activity induced by NDP p.C126F [21]. As for NDP p.R107Pfs and NDP p.L108Cfs, the subsequent amino acids' substitutions might imply the conformational changes of the $\beta$ sheet of Norrin and dimerization, which still need further investigation to clarify. However, there are some discrepancies between the binding defects of FZD4 M105V to Norrin, located at the same position as FZD4 p.M105R and reported by Xu et al. and Qin et al., where the former describe a normal production at plasma membrane and the latter detect obvious binding defects $[13,18]$.

So far, there have been at least nine genes attributed to the progression of FEVR including NDP [26], FZD4 [27], LRP5 [28], TSPAN12 [29], ZNF408 [30], KIF11 [31], RCBTB1 [32], CTNNB1 [33], and JAG1 [34]. Our previous results also provided insight into the relationship between a novel splicing mutation (c.734+1G>A) in CTNNB1 and a 27-year-old Chinese pregnant woman with a severe intellectual disability and FEVR [35]. Human FEVR displays variable ocular defects and serves as an excellent model to explore Wnt signaling, especially causing mutations in NDP, FZD4, LRP5, and TSPAN12. To date, the NorrinFrizzled-4 signaling system has been clarified to play a central role in retinal vascular development and blood-brain barrier plasticity $[13,36]$. Luhmann et al. showed that $N D P$-knockout mice could occur in the regression of the hyaloid vascular alongside the delay of the superficial retinal vasculature and the failure of the deep retinal vasculature [37]. Similarly, FZD4-knockout mice have been shown defective in vasculature in the retina and inner ear [13]. Xia et al. verify that LRP5-knockout mice develop capillary network incompletely and capillaries lacking lumen structure [38]. Afterwards, the formation of microaneurysms, aberrant fenestration, and delayed hyaloid vessel regression could be observed in the TSPAN12-knockout mice [12]. Subsequently, further research on the role of Norrin/ $\beta$-catenin in the retinal vascular induced by the mutations detected in our study would be carried out using animal models. 


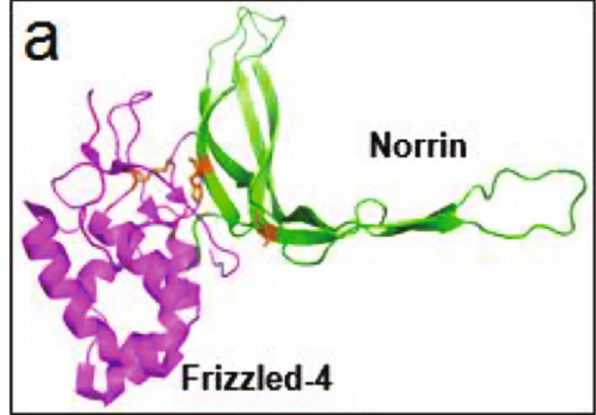

(a)

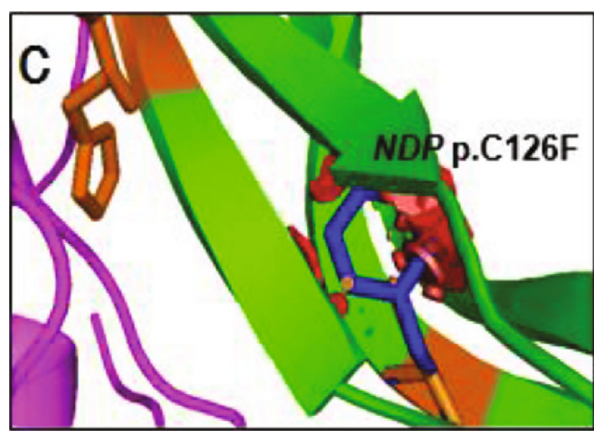

(c)

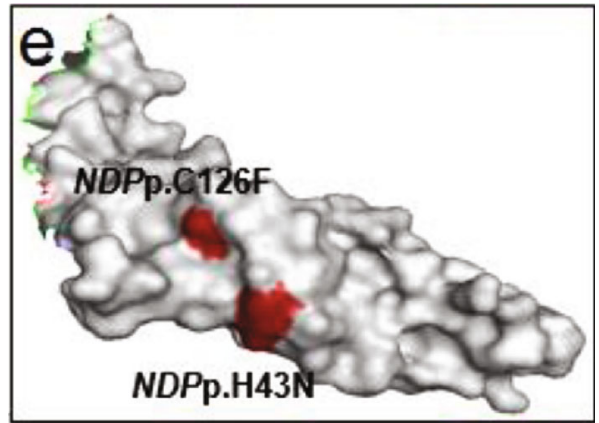

(e)

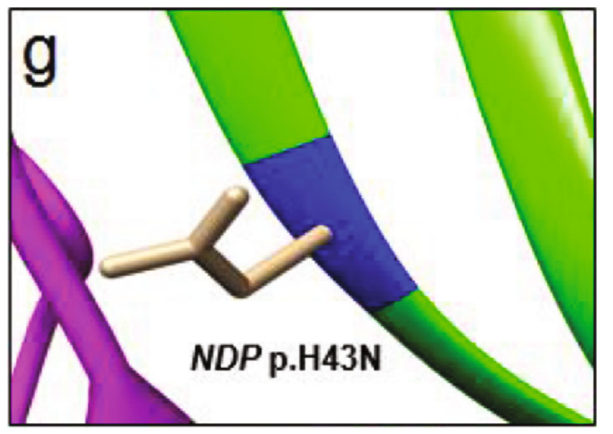

(g)

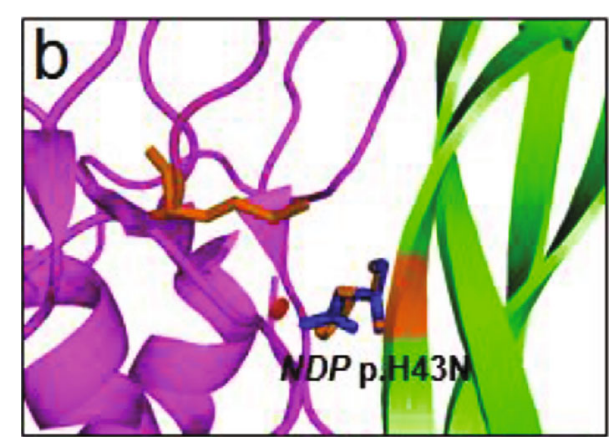

(b)

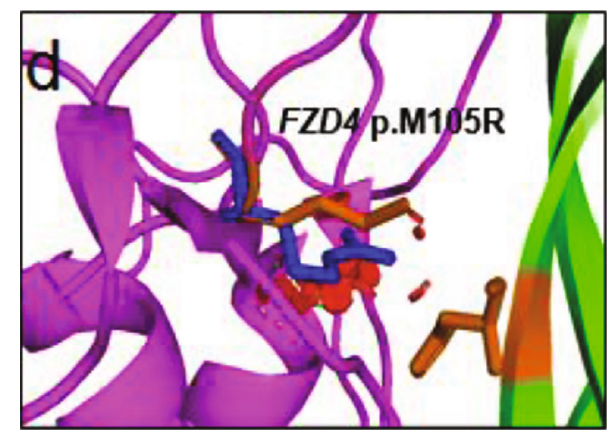

(d)

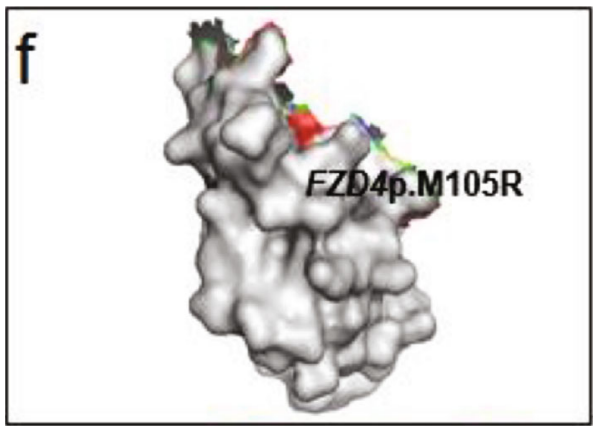

(f)

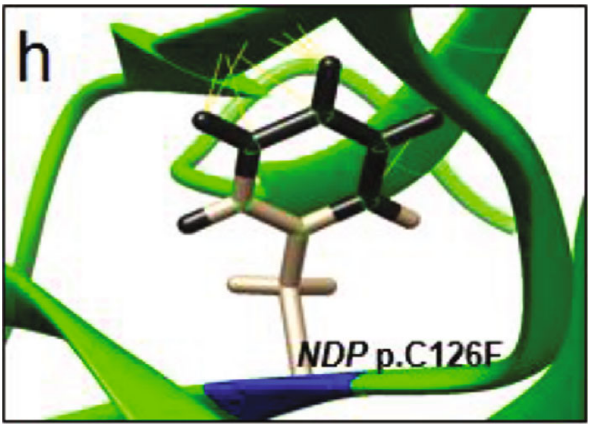

(h)

Figure 2: Continued. 


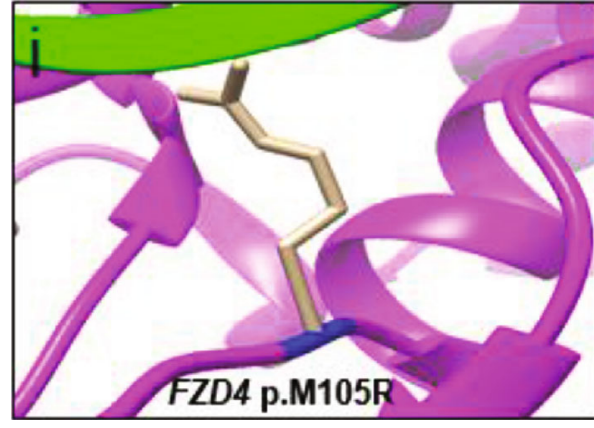

(i)

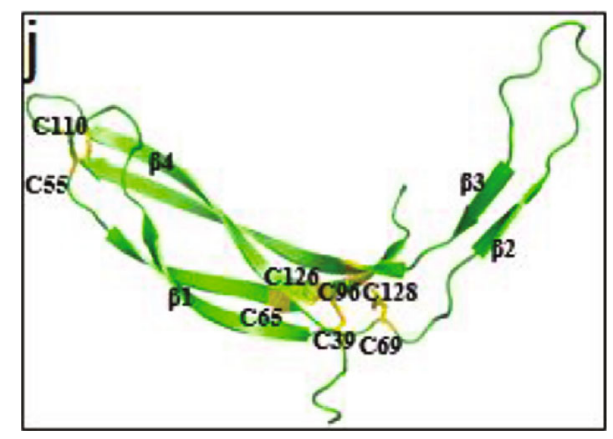

(j)

FIGURE 2: 3D modeling of NDP and FZD4 missense changes. (a) Ribbon model of Norrin and Frizzled-4. The wild-type residues of NDP and FZD4 missense mutations were depicted in orange. (b-d) Closeup of NDP p.H43N, NDP p.C126F, and FZD4 p.M105R. The most stable confirmation was represented in blue. (e, f) NDP and FZD4 missense mutations were located at the protein interface. (g) After minimizing the protein energy, NDP p.H43N could not produce clash/contact bonds. (h) After minimizing the protein energy, NDP p.C126F produced clash/contact bonds, which were shown in yellow. (i) After minimizing the protein energy, FZD4 p.M105R could not produce clash/contact bonds. (j) The cysteine side chains that participated in forming disulfide bonds and creating the critical knot motif were shown in yellow.

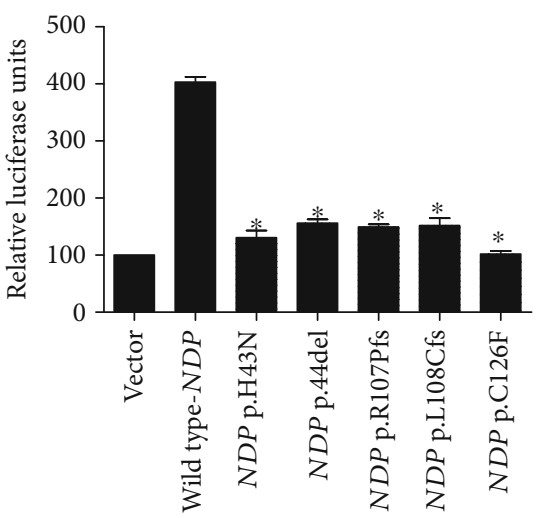

(a)

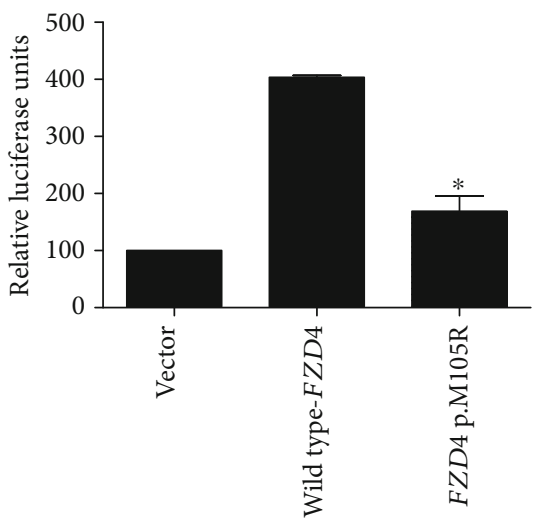

(b)

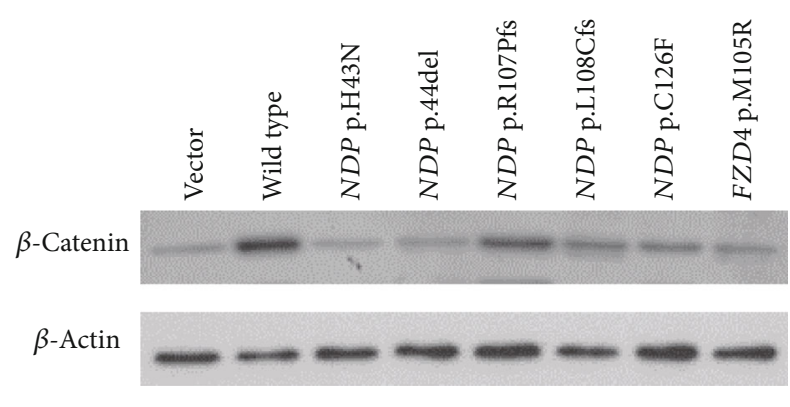

(c)

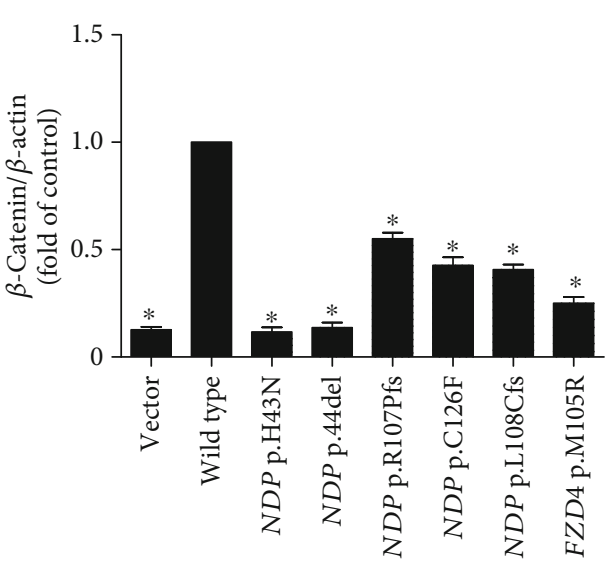

(d)

Figure 3: Topflash reporter assay and Western blotting. (a, b) Failure of NDP and FZD4 mutants in the activation of Norrin/ $\beta$-catenin signaling $(72.5 \%$ reduction for NDP p.H43N, 70\% reduction for NDP p.44del, $71.2 \%$ reduction for NDP p.R107Pfs, $70.5 \%$ reduction for NDP p.L108Cfs, 73\% reduction for NDP p.C126F, and 65\% reduction for FZD4 p.M105R). The experiment was carried out in triplicate at the same time and repeated three times. (c, d) Western blotting showed the expression level of $\beta$-catenin was decreased, similar to the Topflash reporter assay. Asterisks indicated significant differences from the positive control by pairwise Student's test $(P<0.05)$. 


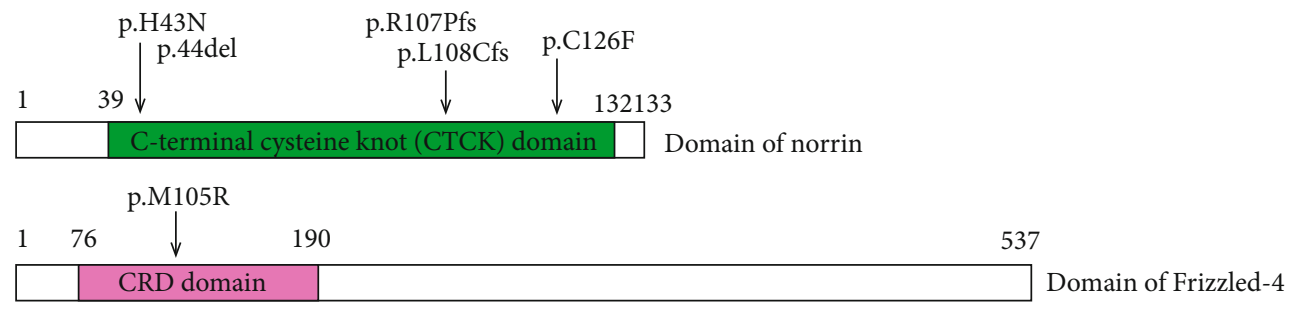

(a)

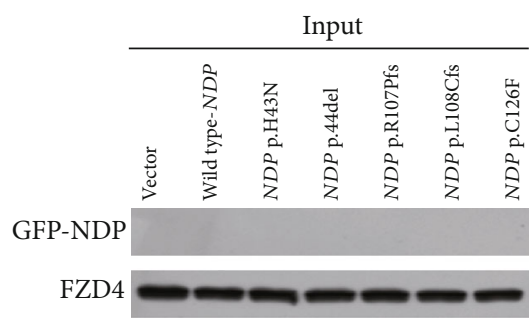

(b)

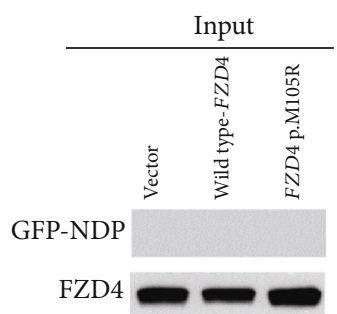

(d)

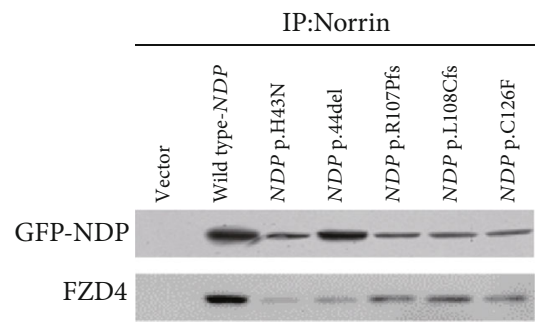

(c)

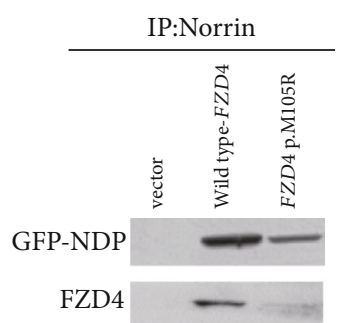

(e)

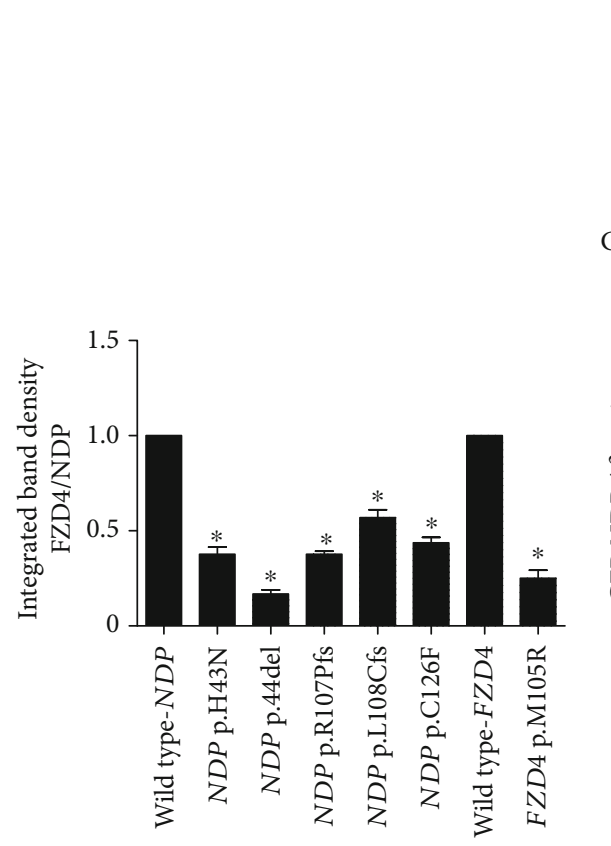

(f)

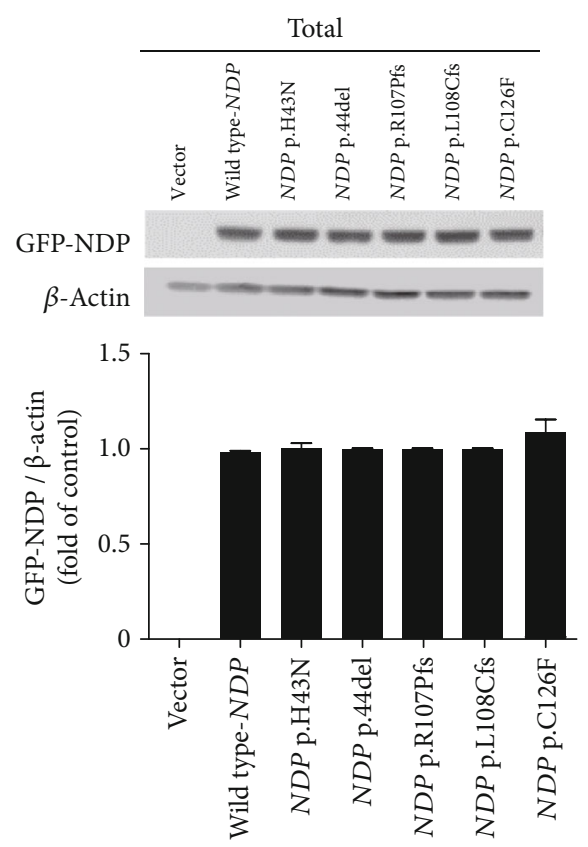

(g)

FIgure 4: Continued 

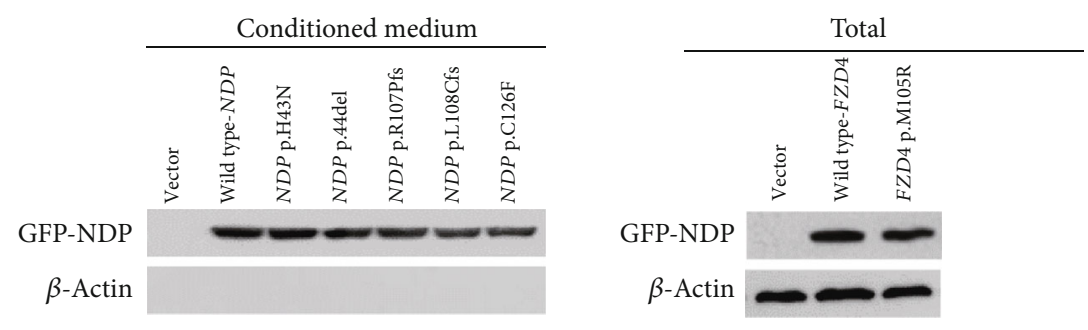

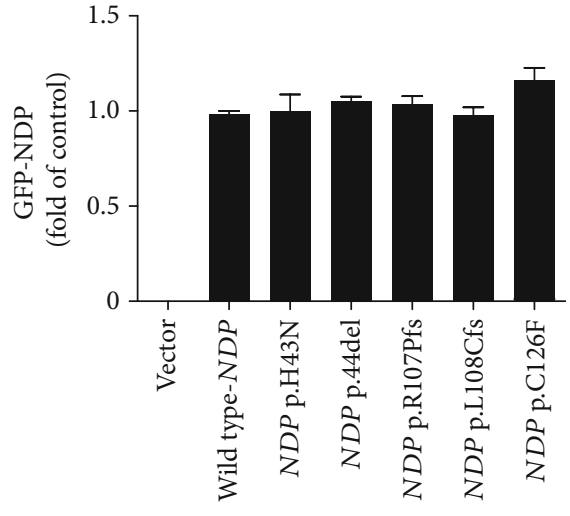

(h)

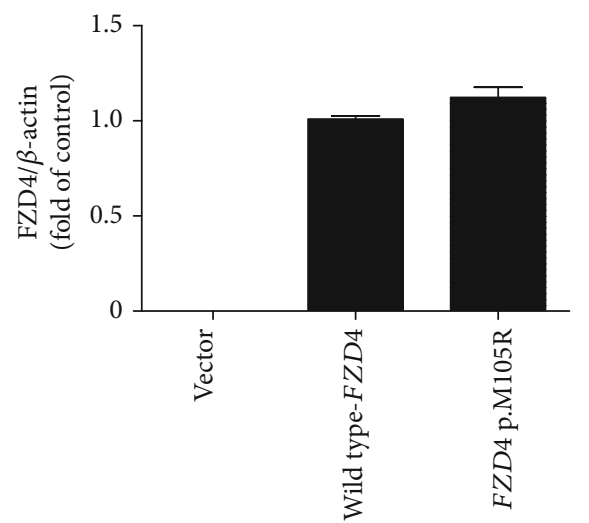

(i)
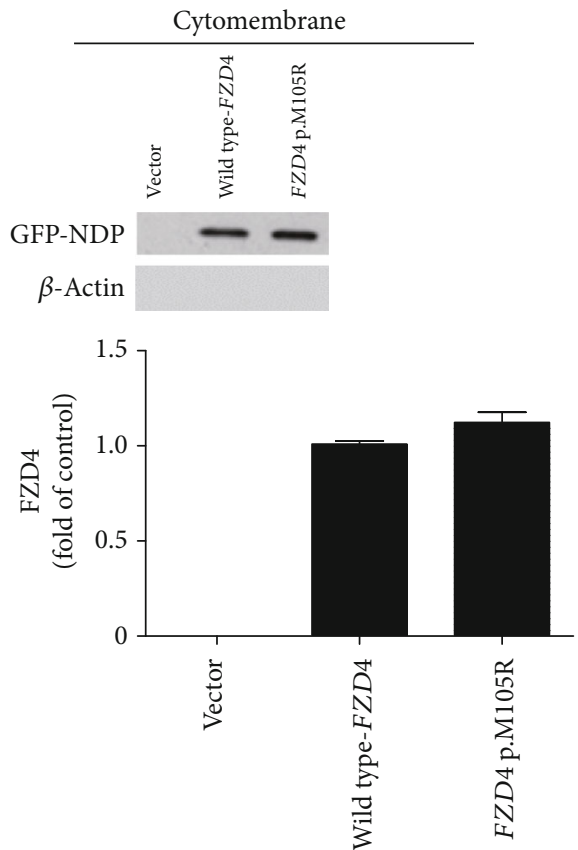

(j)

FIGURE 4: FEVR-linked NDP and FZD4 mutations impaired the binding of Norrin and Frizzled-4. (a) The schematic diagram showed that all the six mutations were located in the CTCK or CRD domain, respectively. (b, c) Transfected cells expressing Frizzled-4 were incubated with wild-type and mutant Norrin-conditioned medium before the Norrin-Frizzled-4 binding complex was immunoprecipitated. Mutant Norrin coprecipitated with Frizzled-4 to varying reduced degrees. It was noted that Norrin concentrations in the diluted lysate were below the detection limit of standard detection systems. (d, e) Transfected cells expressing wild-type and mutant Frizzled-4 were incubated with Norrin-conditioned medium before the Norrin-Frizzled-4 binding complex was immunoprecipitated. Norrin coprecipitated with mutant Frizzled-4 to a distinct reduced degree. It was noted that Norrin concentrations in the diluted lysate were below the detection limit of standard detection systems. (f) Integrated band density quantification of Frizzled-4 coprecipitated with Norrin. (g) Mutant Norrin was expressed at the same level as wild-type Norrin. (h) The secretion of extracellular mutant Norrin was expressed at the same level as wildtype Norrin. (i) Mutant Frizzled-4 was expressed at the same level as wild-type Frizzled-4. (j) Mutant Frizzled-4 was efficiently located to the plasma membrane the same as wild-type Frizzled-4. 


\section{Conclusions}

Pathogenic mutations in NDP and FZD4 could lead to a series of retina-related diseases such as FEVR, Norrie, and retinopathy of prematurity (MIM\#133780), which could be distinguished according to their unique characteristics. Furthermore, our present work represents a step in representing points along the FEVR phenotypic spectrum. Taken together, these findings would allow a reliable assessment of FEVR recurrence and improve genetic counseling. We believe that our findings would provide essential evidence for the follow-up study of animal models and drug targets, as well as for understanding the underlying molecular mechanism of FEVR [39].

\section{Data Availability}

The data used to support the findings of this study are included within the article.

\section{Conflicts of Interest}

The authors declare that they have no conflicts of interest regarding the publication of this article.

\section{Authors' Contributions}

Shuai Han, Junhui Sun and Liwei Yang contributed equally to this work.

\section{Acknowledgments}

The authors would like to thank the patients and their families for their participation and cooperation. The study was partially supported by the grant from the National Natural Science Foundation of China (31371271) and the Natural Science Foundation of Zhejiang Province (LZ14C060001).

\section{Supplementary Materials}

Figure S1: Norrin and Frizzled-4 sequence alignment of missense mutations. (Supplementary Materials)

\section{References}

[1] V. G. Criswick and C. L. Schepens, "Familial exudative vitreoretinopathy," American Journal of Ophthalmology, vol. 68, no. 4, pp. 578-594, 1969.

[2] T. M. Ranchod, L. Y. Ho, K. A. Drenser, A. Capone Jr., and M. T. Trese, "Clinical presentation of familial exudative vitreoretinopathy," Ophthalmology, vol. 118, no. 10, pp. 2070-2075, 2011.

[3] C. L. B. Canny and G. L. Oliver, "Fluorescein angiographic findings in familial exudative vitreoretinopathy," Archives of Ophthalmology, vol. 94, no. 7, pp. 1114-1120, 1976.

[4] J. M. Robitaille, K. Wallace, B. Zheng et al., "Phenotypic overlap of familial exudative vitreoretinopathy (FEVR) with persistent fetal vasculature (PFV) caused by FZD4 mutations in two distinct pedigrees," Ophthalmic Genetics, vol. 30, no. 1, pp. 2330, 2009 .
[5] W. Berger, B. Kloeckener-Gruissem, and J. Neidhardt, "The molecular basis of human retinal and vitreoretinal diseases," Progress in Retinal and Eye Research, vol. 29, no. 5, pp. 335375, 2010.

[6] K. Nikopoulos, H. Venselaar, R. W. J. Collin et al., "Overview of the mutation spectrum in familial exudative vitreoretinopathy and Norrie disease with identification of 21 novel variants in FZD4, LRP5, and NDP," Human Mutation, vol. 31, no. 6, pp. 656-666, 2010.

[7] D. F. Gilmour, "Familial exudative vitreoretinopathy and related retinopathies," Eye, vol. 29, no. 1, pp. 1-14, 2015.

[8] M. Gal, E. Y. Levanon, Y. Hujeirat, M. Khayat, J. Pe'er, and S. Shalev, "Novel mutation in TSPAN12 leads to autosomal recessive inheritance of congenital vitreoretinal disease with intra-familial phenotypic variability," American Journal of Medical Genetics Part A, vol. 164, no. 12, pp. 2996-3002, 2014.

[9] A. H. Kashani, K. T. Brown, E. Chang, K. A. Drenser, A. Capone, and M. T. Trese, "Diversity of retinal vascular anomalies in patients with familial exudative vitreoretinopathy," Ophthalmology, vol. 121, no. 11, pp. 2220-2227, 2014.

[10] Z. Wang, C. H. Liu, S. Huang, and J. Chen, "Wnt signaling in vascular eye diseases," Progress in Retinal and Eye Research, vol. 70, pp. 110-133, 2019.

[11] X. He, M. Semenov, K. Tamai, and X. Zeng, "LDL receptorrelated proteins 5 and 6 in Wnt/beta-catenin signaling: arrows point the way," Development, vol. 131, no. 8, pp. 1663-1677, 2004.

[12] H. J. Junge, S. Yang, J. B. Burton et al., "TSPAN12 regulates retinal vascular development by promoting Norrin- but not Wntinduced FZD4/beta-catenin signaling," Cell, vol. 139, no. 2, pp. 299-311, 2009.

[13] Q. Xu, Y. Wang, A. Dabdoub et al., "Vascular development in the retina and inner ear: control by Norrin and Frizzled-4, a high-affinity ligand-receptor pair," Cell, vol. 116, no. 6, pp. 883-895, 2004.

[14] M. B. Lai, C. Zhang, J. Shi et al., "TSPAN12 is a Norrin coreceptor that amplifies frizzled 4 ligand selectivity and signaling," Cell Reports, vol. 19, no. 13, pp. 2809-2822, 2017.

[15] A. M. Goodwin, K. M. Sullivan, and P. A. D'Amore, "Cultured endothelial cells display endogenous activation of the canonical Wnt signaling pathway and express multiple ligands, receptors, and secreted modulators of Wnt signaling," Developmental Dynamics, vol. 235, no. 11, pp. 3110-3120, 2006.

[16] P. J. Southern and P. Berg, "Transformation of mammalian cells to antibiotic resistance with a bacterial gene under control of the SV40 early region promoter," Journal of Molecular and Applied Genetics, vol. 1, no. 4, pp. 327-341, 1982.

[17] S. Han, B. Zhao, X. Pan et al., "Estrogen receptor variant ER- $\alpha 36$ is involved in estrogen neuroprotection against oxidative toxicity," Neuroscience, vol. 310, no. 11, pp. 224-241, 2015.

[18] M. Qin, H. Kondo, T. Tahira, and K. Hayashi, "Moderate reduction of Norrin signaling activity associated with the causative missense mutations identified in patients with familial exudative vitreoretinopathy," Human Genetics, vol. 122, no. 6, pp. 615-623, 2008.

[19] F. Q. Rao, X. B. Cai, F. F. Cheng et al., "Mutations inLRP5,FZD4,TSPAN12,NDP,ZNF408, orKIF11Genes account for $38.7 \%$ of Chinese patients with familial exudative vitreoretinopathy," Investigative Ophthalmology \& Visual Science, vol. 58, no. 5, pp. 2623-2629, 2017. 
[20] S. Richards, N. Aziz, S. Bale et al., "Standards and guidelines for the interpretation of sequence variants: a joint consensus recommendation of the American College of Medical Genetics and Genomics and the Association for Molecular Pathology," Genetics in Medicine, vol. 17, no. 5, pp. 405-424, 2015.

[21] P. M. Smallwood, J. Williams, Q. Xu, D. J. Leahy, and J. Nathans, "Mutational analysis of Norrin-Frizzled 4 recognition," Journal of Biological Chemistry, vol. 282, no. 6, pp. 40574068, 2007.

[22] K. Zhang, Y. Harada, X. Wei et al., "An essential role of the cysteine-rich domain of FZD4 in Norrin/Wnt signaling and familial exudative vitreoretinopathy," Journal of Biological Chemistry, vol. 286, no. 12, pp. 10210-10215, 2011.

[23] P. Fei, X. Zhu, Z. Jiang et al., "Identification and functional analysis of novel FZD4 mutations in Han Chinese with familial exudative vitreoretinopathy," Scientific Reports, vol. 5, no. 1, article 16120, 2015.

[24] G. Royer, S. Hanein, V. Raclin et al., "NDP gene mutations in 14 French families with Norrie disease," Human Mutation, vol. 22, no. 6, p. 499, 2003.

[25] J. L. Dickinson, M. M. Sale, A. Passmore et al., "Mutations in the NDP gene: contribution to Norrie disease, familial exudative vitreoretinopathy and retinopathy of prematurity," Clinical and Experimental Ophthalmology, vol. 34, no. 7, pp. 682-688, 2006.

[26] Z. Y. Chen, E. M. Battinelli, A. Fielder et al., "A mutation in the Norrie disease gene (NDP) associated with X linked familial exudative vitreoretinopathy," Nature Genetics, vol. 5, no. 2, pp. 180-183, 1993.

[27] J. Robitaille, M. L. E. MacDonald, A. Kaykas et al., "Mutant frizzled-4 disrupts retinal angiogenesis in familial exudative vitreoretinopathy," Nature Genetics, vol. 32, no. 2, pp. 326330, 2002.

[28] X. Jiao, V. Ventruto, M. T. Trese, B. S. Shastry, and J. F. Hejtmancik, "Autosomal recessive familial exudative vitreoretinopathy is associated with mutations in LRP5," American Journal of Human Genetics, vol. 75, no. 5, pp. 878-884, 2004.

[29] J. A. Poulter, M. Ali, D. F. Gilmour et al., "Mutations in TSPAN12 cause autosomal-dominant familial exudative vitreoretinopathy," American Journal of Human Genetics, vol. 98, no. 3, p. 592, 2016.

[30] R. W. J. Collin, K. Nikopoulos, M. Dona et al., "ZNF408 is mutated in familial exudative vitreoretinopathy and is crucial for the development of zebrafish retinal vasculature," Proceedings of the National Academy of Sciences of the United States of America, vol. 110, no. 24, pp. 9856-9861, 2013.

[31] J. M. Robitaille, R. M. Gillett, M. A. LeBlanc et al., "Phenotypic overlap between familial exudative vitreoretinopathy and microcephaly, lymphedema, and chorioretinal dysplasia caused by kif11 mutations," JAMA Ophthalmology, vol. 132, no. 12, pp. 1393-1399, 2014.

[32] J. H. Wu, J. H. Liu, Y. C. Ko et al., "Haploinsufficiency of RCBTB1 is associated with Coats disease and familial exudative vitreoretinopathy," Human Molecular Genetics, vol. 25, no. 8, pp. 1637-1647, 2016.

[33] M. W. Dixon, M. S. Stem, J. L. Schuette, C. E. Keegan, and C. G. Besirli, "CTNNB1 mutation associated with familial exudative vitreoretinopathy (FEVR) phenotype," Ophthalmic Genetics, vol. 37, no. 4, pp. 468-470, 2016.

[34] L. Zhang, X. Zhang, H. Xu et al., "Exome sequencing revealed Notch ligand JAG1 as a novel candidate gene for familial exu- dative vitreoretinopathy," Genetics in Medicine, vol. 22, no. 1, pp. 77-84, 2019.

[35] H. Wang, Y. Zhao, L. Yang, S. Han, and M. Qi, "Identification of a novel splice mutation in CTNNB1 gene in a Chinese family with both severe intellectual disability and serious visual defects," Neurological Sciences, vol. 40, no. 8, pp. 1701-1704, 2019.

[36] Y. Wang, A. Rattner, Y. Zhou, J. Williams, P. M. Smallwood, and J. Nathans, "Norrin/Frizzled4 signaling in retinal vascular development and blood brain barrier plasticity," Cell, vol. 151, no. 6, pp. 1332-1344, 2012.

[37] U. F. O. Luhmann, J. Lin, N. Acar et al., "Role of the Norrie disease pseudoglioma gene in sprouting angiogenesis during development of the retinal vasculature," Investigative Ophthalmology and Visual Science, vol. 46, no. 9, pp. 3372-3382, 2005.

[38] C. H. Xia, H. Liu, D. Cheung et al., "A model for familial exudative vitreoretinopathy caused by LPR5 mutations," Human Molecular Genetics, vol. 17, no. 11, pp. 1605-1612, 2008.

[39] R. M. Milhem and B. R. Ali, "Disorders of FZ-CRD; insights towards FZ-CRD folding and therapeutic landscape," Molecular Medicine, vol. 26, no. 1, p. 4, 2019. 